\title{
Personality Styles: Why They Matter in the Workplace
}

\section{Melissa Taylor*}

\section{Abstract}

Personality style is a popular topic. Extroversion has historically been celebrated while society has tried to cure introversion. Most individuals fall somewhere in the middle of the personality spectrum and possess both strengths and weaknesses related to their preference. A variety of personality styles are represented in every workplace and these can cause tension and conflict. Understanding the nuances associated with varying personality styles will allow organizations to accommodate the needs of individual employees leading to greater workplace productivity.

Keywords: Personality Style, Extrovert, Introvert, Extroversion, Introversion

JEL Code: $M$ - Business Administration and Business Economics; Marketing; Accounting; Personnel Economics

Each day individuals come into contact

Eith a variety of personalities (Cooper, 2013). Everyone has an underlying personality disposition, fundamental in patterns of behavior (Sackett \& Walmsley, 2014; SadlerSmith \& Badger, 1998). Personality style begins biologically and is influenced by environment (Opt \& Loffredo, 2000). No personality style is superior (Schmidt, 2016); however, an individual's personality influences others, and personality style differences can create tension and misunderstanding (Varvel, Adams, Pridie, \& Ulloa, 2004).

Personality style is better described as an orientation rather than a choice (Rauch, 2003). Genetics influence the direction individuals lean (Cooper, 2013), and personality style typically remains stable throughout life (Bernstein, 2015; Sadler-Smith \& Badger, 1998). Basic personality tendencies are present at birth and develop over time (Bernstein, 2015; Glass, Li, \& Pan, 2014), typically stabilizing during teenage years (Stewart, Fritz, \& Norwood, 2004) but shifts are possible during an individual's lifetime (Gordon, 2016). How personality styles develop is related to how individuals process information and come to conclusions (Opt \& Loffredo, 2000). Literature supports the idea changes in personality style are possible (Sackett \& Walmsley, 2014) but difficult (Bernstein, 2015).

The most common labels used when describing personality style are introversion and extroversion (Hvidsten, 2016). They are described in many ways including a dimension of personality (Cullen-Lester, Leroy, Gerbasi, \& Nishii, 2016), basic personality categories (Hvidsten, 2016), where energy is obtained (Varvel et al., 2004), individuals' response to stimulation (Dossey, 2016), attitudes of consciousness (Hvidsten, 2016), and the

* Unit Business Manager Intermediate and Doctoral Candidate in Human Resource Development University of Louisville 2100 South Floyd Street, Louisville, Kentucky 40208 


\section{Articles}

extent of socialness, cheerfulness, and talkativeness (Moore \& McElroy, 2012).

It is often believed individuals are one personality style or the other (Cooper, 2013); however, there are not only two options (DiSalvo, 2013). Though most experts focus on the extremes (Bernstein, 2015), personality style is not an absolute (Gordon, 2016) but a spectrum (Bernstein, 2015; Gordon, 2016; Schmidt, 2016) with the introvert and extrovert labels as the extreme ends of the scale (Cooper, 2013). Most individuals are not strictly one personality style (Hvidsten, 2016; Schmidt, 2016), but fall somewhere in the middle of the spectrum (Cooper, 2013). No individual completely fits the description of an introvert or extrovert (Kuofie, StephensCraig, \& Dool, 2015). Someone with a slight preference for introversion will behave differently than someone with a strong preference (Varvel et al., 2004). Personality style is a preference (Farrell, 2017), and while individuals tend to prefer one over another, everyone possesses both introverted and extroverted traits (Hvidsten, 2016). Individuals can overcome their preference when the situation demands (Farrell, 2017).

\section{Purpose}

Many have studied personality style and workplace behavior (Hvidsten, 2016). A great deal of leadership advice is available, but it lacks consistency (O'Brochta, 2014). There are few empirical studies on the link between personality style and job context (Huang et al., 2016). While some authors provide implications for organizations of personality style, (O'Brochta, 2014; Sadler-Smith \& Badger, 1998), many simply provide descriptions of introverts and extroverts (Abrams, 2017; Gordon, 2016). Other authors claim science supports their comments; however, no source is cited (Grant, Gino, \& Hofmann, 2010; Grant, 2017). The method used for this study is a review of literature. The study's purpose is to synthesize available information from a variety of sources, compiling it into specific action steps organizations may use to successfully navigate an organization comprised of a variety of personality styles. The research question this study strives to answer is what steps may be taken by organizations to ensure employees of all personality styles have the ability to contribute their best work and live up to their full potential in the workplace.

\section{Definition of Key Words}

Terms used in this paper are present in other contexts. Definitions for the purpose of this study are provided.

There are many available definitions of personality style in literature (Schmidt, 2016) and psychologists define personality style differently than popular culture (Skakoon, 2015). For this study, personality style is based on an individual's sensitivity to stimulation (Grant, 2014) and affects the level of outside stimulation required to function successfully (Schmidt, 2016). Its common labels, introvert and extrovert, refer to the level of external stimulation preferred by an individual (Skakoon, 2015).

The term cognitive style is used in personality style literature (Sadler-Smith \& Badger, 1998; Stewart et al., 2004). It is similarly defined as the way individuals think, solve problems, and relate to others (SadlerSmith \& Badger, 1998). Here, personality style and cognitive style are used interchangeably.

\section{Description of Literature}

Three search strategies were used to gather data for this literature review - online library searches, popular culture searches and snowballing. Online library searches - including personality theory, Carl Jung, introversion, introversion in the workplace, extroversion, and myths about introversion 


\section{Articles}

- were completed in WorldCat and Google Scholar. After articles were discovered, a snowball approach uncovered additional sources cited from the literature. Google Scholar also revealed a number of blogs on the topic of interest. References include 13 empirical studies, 9 conceptual articles, 13 opinion pieces and one book. Most works cited range in age from 2010 to 2017 with five older articles included.

\section{Organization}

This paper is organized in to three major sections. The first provides a background on personality style. The second discusses available literature on introverts, extroverts, and ambiverts. The final portion provides information on each personality style in the workplace and offers suggestions for organizations on creating an environment where all individuals may thrive while working toward organizational goals.

\section{Personality Theory}

Carl Jung introduced personality theory in his book, Psychological Types, in 1923 (Abrams, 2017; Opt \& Loffredo, 2000; Rauch, 2003). He popularized the terms introvert and extrovert (Bernstein, 2015; Cooper, 2013 Dossey, 2016) to describe different attitudes used to direct energy (Farrell, 2017). His book suggests individuals are born with certain temperamental traits (Opt \& Loffredo, 2000), which has since been supported by research (Grant, 2017). Jung's definition of personality style is the most commonly used and researched (Opt \& Loffredo, 2000). Jung studied the way each personality style views the world (Kuofie et al., 2015) and discovered they process ideas differently (Hvidsten, 2016). His research made a clear distinction between introversion and extroversion (Kuofie et al., 2015). Jung defined introverts as focusing on the inner world and extroverts as focusing on the outer world (Opt \& Loffredo, 2000). He researched the conflicts between the two personality styles (Kuofie et al., 2015), but refrained from endorsing one over the other (Dossey, 2016). This study uses Jung's personality theory as a foundation.

It is beneficial for individuals to know their personality style (Gordon, 2016). While many individuals can sense their preference (Kuofie et al., 2015), various personality instruments based on Jung's research are available to aid individuals in confirming their personality style (Bakan \& Leckart, 1966; Dossey, 2016; Sackett \& Walmsley, 2014; Sadler-Smith \& Badger, 1998). The two most popular personality measures in literature are the Myers-Briggs Type Indicator (Dossey, 2016; O'Brochta, 2014; Rauch, 2003; Varvel et al., 2004) and the Five Factor Model (Glass et al., 2014; Hvidsten, 2016; Moore \& McElroy, 2012; Sackett \& Walmsley, 2014; Varvel et al., 2004). Other measures mentioned in research include the Maudsley Personality Inventory (Bakan \& Leckart, 1966) and the 16 Personality Factor Questionnaire (Sadler-Smith \& Badger, 1998). Most personality instruments measure extroversion/introversion (Hvidsten, 2016).

Personality style tests help organizations identify applicants who will fit with the culture and match individuals with appropriate mentors and clients (Gordon, 2016). It is important to remember even the most popular personality measures are never completely accurate for all people (Kuofie et al., 2015). Personality style tests are highly nuanced and do not measure individual ability or motivation; however, they can help individuals determine fit in a field of study or work (Gordon, 2016).

\section{Personality Styles}

All people are wired differently (Grant, 2017; Walter, 2013). Personality style is related to where individuals gain and restore energy (Vien, 2016); however, the meanings of introvert 


\section{Articles}

and extrovert are often confused (Cooper, 2013). Some believe traditional definitions of personality style are myths (Grant, 2014) because many personality stereotypes are incorrect (DiSalvo, 2013). Personality style is not the same as cognitive capacity (SadlerSmith \& Badger, 1998) and oversimplified definitions are insufficient (Grant, 2014). The true difference between personality styles relates to how an individual's mind and body responds to stimulation (Cooper, 2013). The brains of introverts and extroverts process information differently (Cooper, 2013), and personality style connects cognition and personality (Stewart et al., 2004). Style preference affects an individual's approach to socializing (Schmidt, 2016), social media usage (Moore \& McElroy, 2012), stress (Gordon, 2016), and work habits (Schmidt, 2016).

Personality style is a continuum without a clear defining line (Korn, 2017). Most fall somewhere in the middle of the scale (Cooper, 2013) and have attributes of both introversion and extroversion (Abrams, 2017; Gordon, 2016). Style preference leads to behavioral patterns (Glass et al., 2014); however, extroverts and introverts can still do things associated with the other style (Cooper, 2013). Everyone has a dominate personality style (Abrams, 2017), but possesses aspects of both (Kuofie et al., 2015). An individual may not be able to change style preference, but can change behavior patterns (Sackett \& Walmsley, 2014). Personality style is similar to being right or left handed; everyone has a preference, but can use both (Cooper, 2013; Kuofie et al., 2015).

\section{Extroverts}

Most individuals lean toward the extroversion end of the personality spectrum (Gordon, 2016). Extroversion refers to a person's social ability and outgoingness
(Glass et al., 2014). Society has a cultural bias toward extroversion (Gordon, 2016; Vien, 2016), and it is often associated with positive attributes (Abrams, 2017). The world is an extroverted culture (Skakoon, 2015) and this busy, noisy culture is an extrovert factory (Dossey, 2016). Individuals learn to be extroverted because it is expected (Gordon, 2016). Society considers extroverted traits ideal (Cain, 2012; Korn, 2017; Schmidt, 2016; Skakoon, 2015) and desirable for success (Grant, 2017; Schmidt, 2016). Being outgoing is normal in today's society, and being called a people-person is a compliment (Rauch, 2003). Outgoing individuals are viewed as better-looking, more intelligent and more interesting (Korn, 2017). The strengths of extroversion are highly valued (Farrell, 2017).

Extroverts naturally attract followers (Kuofie et al., 2015) causing others around them to behave more sociably (Varvel et al., 2004). Collaboration is popular (Dossey, 2016) and extroverts are viewed as natural born leaders (Kuofie et al., 2015). They have contagious energy (Grant, 2013), easily getting others excited about their ideas (Vien, 2016). Energy is transferred between people, and extroverts energize others (Cullen-Lester et al., 2016).

Extroverts are talkative (Abrams, 2017; Cullen-Lester et al., 2016; Grant, 2014; Sackett \& Walmsley, 2014). They enjoy conversing with others (Hvidsten, 2016) and ask a lot of questions (Farrell, 2017). Extroverts are likely to express ideas and concerns (Cullen-Lester et al., 2016). Their loud voices typically get attention (O'Brochta, 2014). Extroverts tend to dominate conversation (Grant, 2014; Grant, 2013) and take over discussions (Grant et al., 2010). Extroverts have strong communication skills (Stewart et al., 2004), prefer talking over listening (Schmidt, 2016) and value the opportunity to share their ideas (Cullen-Lester et al., 2016). They are driven by the constant 


\section{Articles}

stimulation of conversation (Hvidsten, 2016) are comfortable speaking in public (Skakoon, 2015).

The opinions and ideas of extroverts are often made known (Cullen-Lester et al., 2016; Remund, 2015). Extroverts think out loud (Rauch, 2003; Schmidt, 2016), forming thoughts as they speak (Bernstein, 2015; Gordon, 2016). They process information by talking through ideas (Farrell, 2017; Hvidsten, 2016). Extroverts appear eager and excited in group discussion (Schmidt, 2016), instinctively share information (Skakoon, 2015), throw out whatever comes to mind (Bernstein, 2015), and bounce from one conversation to another (Grant, 2013). They give quick responses (Hvidsten, 2016), often talking before thinking (Gordon, 2016). Most focus on driving a conversation rather than what is being said (Hvidsten, 2016).

Extroverts require stimulation from their environment (Abrams, 2017; Cain, 2012; Grant, 2014; Grant, 2017; Schmidt, 2016). They gather energy from the outside world (Varvel et al., 2004) by interacting with other people (Cooper, 2013; Gordon, 2016; Korn, 2017). Extroverts draw energy externally (Bernstein, 2015; Farrell, 2017) and are energized by other people (Cooper, 2013; Korn, 2017; Rauch, 2003; Vien, 2016). They crave company (Cooper, 2013) and constant connection to others (Remund, 2015). Extroverts bring energy to (Cullen-Lester et al., 2016), and are energized by social interaction (Abrams, 2017), both face-to-face and via phone (Gordon, 2016). Social media is an extrovert's paradise (Dossey, 2016).

Most extroverts have strong social skills (Farrell, 2017; Hvidsten, 2016) and seek out people and activity (Bernstein, 2015). They love crowds (Gordon, 2016) and prefer to be around a lot of people (Bernstein, 2015). Extroverts require very little down time (Abrams, 2017; Cain, 2012), prefer not to be
Personality Styles: Why They Matter in the Workplace

by themselves (Schmidt, 2016) and become bored and restless when alone (Bernstein, 2015; Rauch, 2003) or in quiet environments (Gordon, 2016). They wilt (Rauch, 2003) or feel out of sorts (Vien, 2016) when alone for an extended period (Rauch, 2003) and need to recharge after intently focusing on something (Cooper, 2013). Silence can be frightening for extroverts (Dossey, 2016), and they cannot imagine seeking solitude (Rauch, 2003).

Extroverts are not simply social butterflies (Cullen-Lester et al., 2016; Grant, 2017). They are described as active (Glass et al., 2014), outgoing (Cooper, 2013; Cullen-Lester et al., 2016; Hvidsten, 2016; Skakoon, 2015), happy (Rauch, 2003), spontaneous (Hvidsten, 2016), go-getters (Gordon, 2016), charismatic (Dossey, 2016; Grant 2014), bighearted (Rauch, 2003), effusive (Vien, 2016), warm (Stewart et al., 2004; Rauch, 2003), fearless (Kuofie et al., 2015), energetic (Glass et al., 2014), empathetic (Rauch, 2003), friendly (Stewart et al., 2004), gregarious (CullenLester et al., 2016; Vien, 2016), enthusiastic (DiSalvo, 2013; Grant, 2013) and confident (Grant, 2013; Rauch, 2003). They are sociable (Abrams, 2017; Cullen-Lester et al., 2016; Glass et al., 2014; Hvidsten, 2016; Stewart et al., 2004) risk-takers (Cooper, 2013; Farrell, 2017; Schmidt, 2016) who find the unknown appealing (Kuofie et al., 2015).

At times, the benefits of extroversion have diminishing returns (Grant, 2013). Extroverts are also referred to as stubborn (CullenLester et al., 2016), excessively excited (Grant, 2013), self-centered (Farrell, 2017), egotistical (Dossey, 2016), overly assertive (CullenLester et al., 2016; Glass et al., 2014; Gordon, 2016; Grant, 2013; Schmidt, 2016), forceful (Grant, 2013), dominant (Cullen-Lester et al., 2016; Schmidt, 2016), overbearing (Grant, 2014), bossy (Farrell, 2017), aggressive (Dossey, 2016), arrogant (Farrell, 2017), and impulsive (Kuofie et al., 2015). Extroverts 


\section{Articles}

sometimes come on too strong (Grant, 2014) and may be seen as trying to dominate others' perspectives (Cullen-Lester et al., 2016).

Extroverts gravitate toward the spotlight (Grant, 2013). They have an endless appetite for attention (Bernstein, 2015; Grant, 2013; Rauch, 2003), commanding (Farrell, 2017; Gordon, 2016; Grant et al., 2010) and even hogging it (Skakoon, 2015). Extroverts are comfortable with conflict (Gordon, 2016; Schmidt, 2016) and sometimes experience difficult relationships in teams (Grant, 2014). They make emotional decisions (Kuofie et al., 2015), focusing on their own perspectives while unintentionally suppressing the ideas of others (Grant, 2013). Extroverts assume company is always welcome (Rauch, 2003). They may elicit negative reactions when they do not take no for an answer (Grant, 2013). In spite of these negatives, many individuals pretend to be extroverts because it is viewed more favorably (Korn, 2017). However, the extroverted ideal is being reexamined (Kuofie et al., 2015).

\section{Introverts}

Introverts fall on the other end of the personality style spectrum (Abrams, 2017). They are often overlooked (Vien, 2016) and rarely credited for their gifts (Abrams, 2017). Introvert statistics are unclear (Korn, 2017). Though there is disagreement on how many individuals are introverts (Rauch, 2003), most literature agrees it is between one third and one half of the American population (Abrams, 2017; Cain, 2012; Dossey, 2016; Godsey, 2015; Korn, 2017; Kuofie et al., 2015). Defining introversion is complicated (Korn, 2017). Dictionaries describe introverts as shy, quiet, withdrawn, and reserved (Hvidsten, 2016). Despite appearing (Godsey, 2015) and being described (Korn, 2017), perceived (Farrell, 2017), and labeled as shy (Kuofie et al., 2015), not all introverts are (Abrams, 2017;
Cain, 2012; Cooper, 2013; Gordon, 2016; Rauch, 2003; Schmidt, 2016; Skakoon, 2015). Introverts feel most alive in quiet environments (Cain, 2012) and, therefore, prefer quiet places (Godsey, 2015). Some introverts are quiet (Cullen-Lester et al., 2016), but this is often mistaken as truth for all (Cain, 2012; Hvidsten, 2016). Introverts are empathetic and easily relate to the feelings of others (Abrams, 2017; Grant, 2017). They are often intelligent (Rauch, 2003), and a majority of the gifted population are introverts (Dossey, 2016; Rauch, 2003). Introverts enjoy learning simply for the joy it brings (Hvidsten, 2016). Academics are typically introverts (Raza, ulMustafa, \& Capretz, 2012).

Introverts are most comfortable when alone (Bernstein, 2015; Dossey, 2016; Kasriel, 2016; Korn, 2017; Walter, 2013). They need time to unwind and decompress (Gordon, 2016) and view solitude as a sanctuary (Abrams, 2017; Dossey, 2016). Introverts resist outside influences (Bernstein, 2015; Kuofie et al., 2015) and being herded together with others (Cain, 2012). Alone time allows introverts to reenergize (Abrams, 2017; Cooper, 2013; Dossey, 2016; Gordon, 2016; Walter, 2013). Solitude is not trendy (Dossey, 2016) so unfortunately, introverts are often described as withdrawn (Glass et al., 2014; Hvidsten, 2016; Rauch, 2003) and feel guilty for needing space (Cain, 2012).

Introverts are overwhelmed by prolonged social interaction (Grant, 2014) and outside stimulation (Abrams, 2017; Cooper, 2013; Gordon, 2016) including bright lights and loud noises (Grant, 2014). They need moderation in social settings (Remund, 2015) and may be exhausted after large gatherings (Korn, 2017; Kuofie et al., 2015). Introverts find it tiring to be around other people (Bernstein, 2015; Cooper, 2013; Rauch, 2003), and must recharge even after enjoyable social situations (Korn, 2017; Rauch, 2003; Schmidt, 2016). They 


\section{Articles}

gain energy from alone time (Cooper, 2013; Remund, 2015) allowing them to contribute socially again (Vein, 2016). The more an introvert recharges, the more present they are for others (Cain, 2012). Quiet places provide introverts clarity (Dossey, 2016) through an opportunity to reflect on thoughts and feelings (Grant, 2014). Introverts are introspective (Farrell, 2017; Kuofie et al., 2015), have a large capacity for reflection (Abrams, 2017; Vien, 2016), and gather energy from focusing on thoughts and ideas (Gordon, 2016; Hvidsten, 2016; Korn, 2017; Varvel et al., 2004).

Introverts use long-term memory to create complex associations (Schmidt, 2016). They are analytical thinkers (Kuofie et al., 2015; Farrell, 2017; O'Brochta, 2014) with strong problem-solving skills who compare current situations to previous experience and apply developed skills to new tasks (Stewart et al., 2004). Introverts are good problem-solvers (Gordon, 2016; Korn, 2017) considering the long-term impact of their actions (O'Brochta, 2014); however, introverts do not view every situation as a problem requiring a solution (Kasriel, 2016). They are good critical thinkers (Korn, 2017) who process information by consideration rather than discussion (Dossey, 2016). Introverts require time to consider information and will provide detailed responses (Farrell, 2017).

Introverts excel at handling uncertainty (Korn, 2017) because they are intentional (Vien, 2016), consider implications (Remund, 2015), and plan ahead (Abrams, 2017) rather than rushing into decisions (Cain, 2012). Introverts think thoroughly (Grant, 2017) and make careful decisions grounded in research (Vien, 2016). Introverts approach risk deliberately (Gordon, 2016), reflect on new ways of doing things (Abrams, 2017) and assume risks only after careful research (Cain, 2012). They are more likely to problem solve using hypothesis testing (Stewart et al.,
Personality Styles: Why They Matter in the Workplace

2004) and base decisions on facts rather than feelings (O'Brochta, 2014). Introverts want to avoid making the mistakes of others (Kasriel, 2016).

Introverts rarely spark conversation, but participate if invited (Remund, 2015). They have a low threshold for small talk (Abrams, 2017; Rauch, 2003), preferring meaningful conversation with purpose (Abrams, 2017; Remund, 2015). Introverts are typically not chatty (Remund, 2015) and provide their best input when not interrupted (Walter, 2013). They are usually not master communicators (Raza, ul-Mustafa, \& Capretz, 2012) and may appear lacking in interpersonal skills (Glass et al., 2014; Moore \& McElroy, 2012). Introverts prefer one-on-one conversations (Abrams, 2017; Cooper, 2013; Hvidsten, 2016; Remund, 2015) where they are warm, sociable, and friendly and may appear extroverted (Korn, 2017). Introverts prefer to prepare comments in advance (Hvidsten, 2016). They favor processing information through writing or thinking before discussing it with others (Kuofie et al., 2015). Introverts prefer writing over talking (Dossey, 2016; Hvidsten, 2016; Schmidt, 2016; Skakoon, 2015). They enjoy engaging in deep conversation (Schmidt, 2016) with few people (Kasriel, 2016). Introverts like socializing in small groups (Skakoon, 2015), often focusing social energy on close friends and family (Schmidt, 2016). They tend to be good listeners (Gordon, 2016; Hvidsten, 2016; Korn, 2017; Vien, 2016) because they default to listening before talking (Dossey, 2016; Kasriel, 2016; Remund, 2015; Schmidt, 2016). Introverts listen intently (Remund, 2015) allowing them to learn (Farrell, 2017). They are conscientious and want to do things well (Korn, 2017).

Introverts are often highly creative (Korn, 2017; Kuofie et al., 2015). They work slowly and deliberately, preferring to focus on one task at a time (Schmidt, 2016). Introverts 


\section{Articles}

are sensitive to critique (Hvidsten, 2016), but appreciate recognition and acknowledgement (Abrams, 2017). They tend to be modest (Vien, 2016) and willingly give away the spotlight (Abrams, 2017). Introverts demonstrate humility (Farrell, 2017) and usually will not brag on themselves (Dossey, 2016). Selfpromotion does not come naturally for them (Vien, 2016).

While the reserved and humble used to be revered (Abrams, 2017), many definitions now imply a negative view of introversion (Kuofie et al., 2015). There is a stigma of introversion (Grant, 2014), and it is rarely viewed as a positive trait (Korn, 2017). Myths believed about introverts are often untrue, unfair, and cruel (Grant, 2017). Society undervalues introversion and attempts to break introverts out of their comfort zones (Godsey, 2015; Gordon, 2016). Individuals who are not extroverts are taught to believe something is wrong with them (Cain, 2012). Introverts do not need to be fixed (Dossey, 2016), medicated (Rauch, 2003), or cured (Dossey, 2016, Schmidt, 2016). In the past, introverts were even diagnosed as schizoid (Kuofie et al., 2015). Introversion is often viewed as a liability (Grant, 2014; Huang et al., 2016), and society attempts to help introverts overcome their shortcomings (Cain, 2012). Because introversion is viewed as a character flaw (Dossey, 2016) many introverts deny their personality style preference (Rauch, 2003).

Introversion is viewed as the negative end of the personality style spectrum (Abrams, 2017). Introverts are wildly misunderstood (Rauch, 2003) and frequently given a bad rap (Abrams, 2017). Introverts are assumed to be reserved (Grant, 2014; Rauch, 2003; Skakoon, 2015) and aloof (Farrell, 2017; Grant, 2014; Rauch, 2003, Remund, 2015; Stewart et al., 2004). Because introverts are careful decision makers (Kuofie et al., 2015), they are viewed as slow and indecisive
(Farrell, 2017). Introverts have been described as conceited (Remund, 2015), depressed (Godsey, 2015), loners (Grant, 2017), timid (Rauch, 2003), antisocial (Abrams, 2017; Godsey, 2015), odd (Kuofie et al., 2015), pessimistic (Rauch, 2003), unenthusiastic (Schmidt, 2016) disinterested (Abrams, 2017; Vien, 2016), annoying (Dossey, 2016) and rude (Grant, 2017). Introverts have also been mistaken as haughty (Rauch, 2003), avoidant (Grant, 2017), arrogant (Gordon, 2016), and bored (Abrams, 2017). With these many misconceptions (Rauch, 2003) introverts have to overcome a strong cultural bias to be successful (Grant et al., 2010).

Luckily, introversion is being reexamined (Korn, 2017), and its negative perception is evaporating (Grant, 2014). Susan Cain is popular in introversion research (Abrams, 2017; Godsey, 2015; Gordon, 2016; Grant, 2014; Hvidsten, 2016; Kuofie et al., 2015; O'Brochta, 2014; Schmidt, 2016; Skakoon, 2015; Walter, 2013). Her book, Quiet, raises awareness about the strengths of introversion (Godsey, 2015). Recent literature has placed introversion in a more positive light (Korn, 2017) focusing on these strengths (Kuofie et al., 2015). Many introverts are now willing to admit their personality style (Grant, 2014). Popular press is also addressing the complexities of introverted personality traits (Kuofie et al., 2015).

Introverts fit into the world like a square peg in a round hole (Abrams, 2017). Though introverts are common (Rauch, 2003), society has forced them to live in an extroverted world (Farrell, 2017; Kuofie et al., 2015). Introverts have learned to function in extroverted situations (Gordon, 2016) where they feel they do not fit in (Kuofie et al., 2015). Some caution introverts against aligning with the world's expectations (Skakoon, 2015). Being introverted does not mean an individual is bad at extroverted things (Gordon, 2016). 


\section{Articles}

Introverts often compensate by using their less dominant side (Kuofie et al., 2015). They step out of their comfort zones to be extroverted when necessary (Gordon, 2016), but may feel like actors in social situations (Rauch, 2003).

\section{Ambiverts}

Carl Jung identified a third group on the personality style spectrum, but did not label it (Bernstein, 2015). This group falls in the middle of the spectrum (Grant, 2013; Korn, 2017). Psychologists began using the term ambivert to describe them in the 1940s (Bernstein, 2015). Ambiverts are balanced between the extremes (Cooper, 2013) as a solid mix of both introversion and extroversion (Bernstein, 2015). A pure ambivert would be equally introverted and extroverted (DiSalvo, 2013). Most people are believed to be ambiverted rather than introverted or extroverted (Grant, 2014). Ambiverts express traits from both personality styles (DiSalvo, 2013), and sometimes neither is dominant (Bernstein, 2015).

Ambiverts enjoy being around people, but it drains them over time (Cooper, 2013). They are enthusiastic without appearing too confident or overexcited (Grant, 2013). Conversely, ambiverts enjoy solitude, but not for extended periods of time (Cooper, 2013). They alternate levels of assertiveness and enthusiasm (Grant, 2013) and recharge with a mixture of social interaction and alone time (Cooper, 2013). Ambiverts are quiet in some situations and talkative in others (Grant, 2014). Sticking with one personality style too long will tire an ambivert (Bernstein, 2015). They alternate between backstage and the spotlight (Grant, 2014) and may not always be sure what will energize them (Bernstein, 2015).

Ambiverts have balanced personalities (Bernstein, 2015) and engage in a flexible pattern of talking and listening (Grant, 2013). They are socially and emotionally
Personality Styles: Why They Matter in the Workplace

flexible (Bernstein, 2015; Grant, 2014) moving between introverted and extroverted activities with ease (Bernstein, 2015). Ambiverts use varied approaches (Cooper, 2013) to find an appropriate balance in a given situation (Grant, 2013). They possess emotional acuity allowing them to choose behaviors appropriate for the setting (Bernstein, 2015). This provides ambiverts an advantage in some work roles (Bernstein, 2015; Cooper, 2013). While they can adapt to the demands of a specific situation (Grant, 2014), ambiverts sometimes have trouble determining which side of their personality to apply given the circumstances (Bernstein, 2015).

\section{Personality in the Workplace}

Today's workplace demands competent employees (Stewart et al., 2004). Introverts and extroverts have different strengths (Walter, 2013) and both personality styles are needed to balance the weaknesses of one another (Farrell, 2017). Many jobs require working with others (O'Brochta, 2014) to accomplish increasingly complex tasks (Stewart et al., 2004). Social skills are important (Hvidsten, 2016) because any team is going to experience stress and conflict (Farrell, 2017). It is important to observe the personality styles of team members (O'Brochta, 2014). Individuals must find a way to coexist and work together (Sadler-Smith \& Badger, 1998). Having the right personality style in the right role produces the best results (Stewart et al., 2004). Organizations need all types of people (Gordon, 2016) with a balance of introversion and extroversion (Farrell, 2017) assigned to roles for which they are best suited (O'Brochta, 2014). Personality style in the workplace has been widely studied (Sackett \& Walmsley, 2014). Individual-task agreement impacts job performance (Stewart et al., 2004), and individuals are more likely 


\section{Articles}

to be satisfied in positions matching their personality style (Huang et al., 2016).

Leaders set the tone for an organization (Harold \& Holtz, 2015) and leadership skills may be developed and strengthened regardless of personality style (Farrell, 2017). A leader's job requires insight into the needs and feelings of others (Kasriel, 2016) in order to build trust and generate support (O'Brochta, 2014). Both introverts and extroverts can be effective leaders (Dossey, 2016; Grant, 2014; Grant, 2017; Korn, 2017) and also possess negative leadership traits (Farrell, 2017). Different types of employees work best with different types of leaders (Grant, 2014; Kuofie et al., 2015); however, successful leaders can adjust their personality style to meet employee needs (Farrell, 2017). When a situation requires a specific skill or trait, these leaders find ways to achieve it (Sackett \& Walmsley, 2014).

Extroverted employees. Extroverts are often perceived as more effective employees (Grant et al., 2010) and are favored in recruiting and promotional decisions (Dossey, 2016; Grant et al., 2010). Care must be taken to ensure positive interviews by extroverts are not mistaken for positive job fit (Huang et al., 2016). There is correlation between personality traits and job performance (Hvidsten, 2016). A fit between employee attributes and job attributes is preferred (Stewart, Et al., 2004). When extroversion is the focus, value is placed more on the employee's personality than actual knowledge (O'Brochta, 2014). Extroverts can more easily meet demands of some jobs (Huang et al., 2016); however, there are times performance suffers if employees are too extroverted (Grant, 2013).

Workplaces focus heavily on extroverted qualities (Kuofie et al., 2015) favoring extroverted preferences (Cain, 2012). Noisy, busy offices cater to extroverts (Farrell, 2017) who prefer open plans and group work
(Cain, 2012). Extroverts prefer interacting while working (Skakoon, 2015) and enjoy collaborative brainstorming (Bernstein, 2015; Cain, 2012; Hvidsten, 2016; Skakoon, 2015). They are often viewed as positively contributing in team environments (Cullen-Lester et al., 2016) and workplaces frequently involve group work (Hvidsten, 2016). Open workspaces can be counterproductive (Cain, 2012; Skakoon, 2015) partially because extroverts may walk around seeking interaction rather than working (Gordon, 2016).

Extroverted leaders. The common perception of an effective leader is someone possessing extroverted traits (Farrell, 2017; Grant et al., 2010; Kuofie et al., 2015) such as cheerfulness and outgoingness (Kasriel, 2016). Some research proposes extroverts are better equipped for leadership (Grant, 2017). They are more likely to seek and obtain leadership positions (Grant, 2014; Korn, 2017). Most top-level executives are extroverts (Farrell, 2017) and many leaders identify as extroverts (Korn, 2017).

Extroverted traits, such as charisma and charm (Kuofie et al., 2015), are expected in leaders (Dossey, 2016; Farrell, 2017). Extroverts thrive in busy environments (Farrell, 2017) and make quick decisions (Hvidsten, 2016; Schmidt, 2016). They engage in intense networking (Grant, 2014) and become fully alive around others (Rauch, 2003). Extroverts enjoy making new acquaintances and have shallow relationships with many people (Hvidsten, 2016). Extroverted leaders are more successful when leading passive employees (Dossey, 2016) and may feel threatened by proactive ones (Grant et al., 2010).

Introverted employees. Introverts are efficient workaholics (Kasriel, 2016) earning respect through action (Remund, 2015). Introverted employees are good listeners (Vien, 2016), who encourage others (Abrams, 2017) and manage time well (Kasriel, 2016). 


\section{Articles}

Introverts prefer working alone (Farrell, 2017; Kuofie et al., 2015). They need to focus and perform better when working independently (Godsey, 2015). Quiet (Farrell, 2017; Schmidt, 2016), subdued (Godsey, 2015) environments with few interruptions are highly desired (Hvidsten, 2016). Too much light and/or noise is draining for introverts (Cain, 2012), and many view open workspaces as chaos and confusion (Dossey, 2016; Kuofie et al., 2015).

The increasing trend toward group projects is difficult for introverts (Abrams, 2017; Godsey, 2015), and they do not get excited about team-building activities (Kuofie et al., 2015); however, groupwork in moderation is beneficial (Godsey, 2015). Introverts are often overlooked in group discussions (Kuofie et al., 2015). They hesitate to pitch ideas (Grant, 2014) and prefer to be asked their opinion rather than jumping in (Walter, 2013). Introverts want to be fully satisfied with their work before sharing it (Hvidsten, 2016; Skakoon, 2015). Providing an agenda in advance allows them to plan ahead for meetings by taking notes and rehearsing information (Kuofie et al., 2015). Many introverts find networking intimidating (Vien, 2016) and exhausting (Gordon, 2016) so meetings tend to be short (Farrell, 2017). During presentations, introverts may use visual aids to divert attention from themselves (Kuofie et al., 2015).

Introverted leaders. Speaking softly was once viewed as a key to success (Abrams, 2017), but now many view introversion as a barrier to leadership (Grant et al., 2010). Introverts possess good leadership qualities (Vien, 2016), but the value of their strengths is not often understood (Farrell, 2017). The positive qualities of introverted leaders are frequently overlooked (Korn, 2017) and underappreciated (Dossey, 2016). This causes organizations to miss out on new ideas and strategies (Kuofie et al., 2015). Historically, it was assumed introverts were ineffective
Personality Styles: Why They Matter in the Workplace

leaders (Farrell, 2017), and a large percentage of senior executives believed introverts in leadership positions were a liability (Grant, 2014; Korn, 2017). Introverts often do not fit the image of an effective leader (Farrell, 2017); however, in reality, introverts are better leaders in certain situations (Grant et al., 2010). Introverted leaders do not desire the spotlight (Kuofie et al., 2015); but have a place in upper management (Farrell, 2017). Introverts aspiring to be leaders will face challenges, and many prefer to simply start their own companies (Kasriel, 2016). Fortunately, there is growing professional and social acceptance of introversion, and leaders are beginning to admit to being introverts (Grant, 2014). Just as introversion is becoming more popular, the concept of quiet leadership is gaining traction (O’Brochta, 2014).

Introverts have a more reserved leadership style (Vien, 2016) and are not micromanagers (Kasriel, 2016). They demand more of their followers (Remund, 2015) succeeding with proactive employees (Grant et al., 2010; Kuofie et al., 2015) who show initiative (O'Brochta, 2014). Introverted leaders are good at motivating others (Walter, 2013) and empower their employees (Kasriel, 2016). They pull ideas from others (Walter, 2013) by helping teams think critically (Kuofie et al., 2015). Introverted leaders validate subordinate initiative (Grant, 2014) expecting direct reports to follow through (Vien, 2016). Followers of introverted leaders must take responsibility and perform without constant reinforcement (Remund, 2015). The key to quiet leadership is trust (O'Brochta, 2014), and employees tend to trust introverted leaders (Vien, 2016). Introverted leaders are open to suggestions (Grant et al., 2010), allow subordinates to participate in decision making (Farrell, 2017), and ensure everyone's voice is heard (Vien, 2016). 


\section{Articles}

Introverts often bring technical skill to leadership roles (Kasriel, 2016) and are willing to coach and advise others (Vien, 2016). Strengths of introverted leaders include listening skills (Grant et al., 2010; Kuofie et al., 2015; Vien, 2016), relationship building (O'Brochta, 2014; Vien, 2016), and leading by example (O'Brochta, 2014). Leaders require strong communication skills, and the best communicators listen well (Abrams, 2017). Introverted leaders listen carefully to direct reports (Grant, 2014), and employees thrive when heard and appreciated (Farrell, 2017).

\section{Implications for Organizations}

Seven suggestions for organizations to help successfully navigate a workplace of various personality styles were revealed through this study. First, it is imperative to understand no personality style is better than another (Walter, 2013). Second, every individual exhibits traits of both introversion and extroversion (Abrams, 2017; Gordon, 2016), and, third, no one is a pure introvert or extrovert (Dossey, 2016; Kuofie et al., 2015). Next, it is important individuals not only discern their own preference (Cain, 2012), but also consider the preferences of others (Cooper, 2013). Finally, organizations should create flexible workspaces (Godsey, 2015), allow for scheduling creativity (Remund, 2015), and take advantage of the strengths of each personality type for the benefit of the overall organization (Cooper, 2013).

\section{Remember No Personality Style is Superior}

There is no right or wrong personality style preference (Varvel et al., 2004), and organizations must equally value both (Schmidt, 2016). A mix of personality styles is necessary to generate innovative ideas (Sadler-Smith \& Badger, 1998). Introversion has as many benefits as extroversion
(Abrams, 2017). An ongoing emphasis on a single personality style will detrimental to organization success (Sadler-Smith \& Badger, 1998). Maintaining a balance is often more beneficial than focusing on one of the extremes (DiSalvo, 2013). No personality style traits are more valuable than others (Schmidt, 2016), and overvaluing extroverted traits can hurt the self-esteem of introverts (Kuofie et al., 2015).

\section{Understand There is No Pure Introvert or Extrovert}

A single word should never be used to define a unique individual (Cooper, 2013). No pure introvert or extrovert exists (Cooper, 2013; Dossey, 2016; Kuofie et al., 2015). Leaders must be careful not to unfairly ascribe all attributes of one personality style to an employee (Farrell, 2017). Organizations must have an understanding of extroversion and introversion (Gordon, 2016).

\section{Identify Own Personality Style Preference}

Understanding their own personality style is the first step in individuals working together effectively (Cooper, 2013). Individuals knowing (Gordon, 2016) and valuing their own personality style is highly beneficial (Varvel et al., 2004). With this knowledge, individuals can tap into what motivates them (Vien, 2016), and be open about their preferences and needs in the workplace (Walter, 2013).

\section{Consider the Preferences of Others}

Individuals are entitled the needs their personality style requires within reason (Cain, 2012). Organizations must acknowledge differing personality styles to maximize employee potential (Kuofie et al., 2015). Effective leaders are aware of not only their own preferences, but also those of colleagues (Farrell, 2017). Consideration of personality style differences allows individuals to get 


\section{Articles}

along and contribute their best work (Cooper, 2013). Successful employees appreciate differing preferences are equally valuable (Varvel et al., 2004).

Rude and disrespectful behavior is common in the workplace (Harold \& Holtz, 2015). Team coordination is necessary, but not easy (Cullen-Lester et al., 2016). Jung specified introverts and extroverts could not work together successfully unless they attempted to understand one another (Kuofie et al., 2015). It is difficult for an extrovert to understand an introvert (Rauch, 2003). Training on the personality preferences of team members improves communication and trust within teams (Varvel et al., 2004). Effective leaders not only adapt their personality style as the situation requires (Farrell, 2017) but also help employees understand one another better (Varvel et al., 2004). Individuals can behave in ways inconsistent with their dominate personality style in specific situations (SadlerSmith \& Badger, 1998). For example, there are times introverts must be social and visible even if it does not come naturally (Vien, 2016).

Accommodating extroverts in the workplace. While literature consistently notes the value of introversion is overlooked (Skakoon, 2015), it is important to continue valuing the contributions of extroverts (Gordon, 2016). Extroverts enjoy meetings (Farrell, 2017) and the opportunity to discuss ideas (Cooper, 2013). They are more likely to be satisfied in roles with a large amount of social interaction (Huang et al., 2016). Extroverts thrive on attention (Bernstein, 2015) and like to be publicly recognized (Cooper, 2013). They may behave in ways interpreted as uncivil, such as checking email during a meeting (Harold \& Holtz, 2015) or unintentionally suppressing others' ideas (Grant, 2013). Those offended need to consider the intent behind an extrovert's behavior (Harold \& Holtz, 2015). The independence of extroverts
Personality Styles: Why They Matter in the Workplace

should be respected (Cooper, 2013), and they should not be criticized for their feelings (Walter, 2013).

Accommodating introverts in the workplace. Organizations should not try to convert introverts (Cooper, 2013). One of the greatest frustrations experienced by introverts is the undervaluing of their gifts (Abrams, 2017). Introverted traits should be cultivated, valued, and nurtured in the workplace (Schmidt, 2016). Introverts ought not be corrected or embarrassed publically (Cooper, 2013). Organizations must respect an introvert's need for privacy (Cooper, 2013), and when possible, provide them a quiet workspace (Dossey, 2016). Group meetings should be short (Farrell, 2017) with meaningful content (Remund, 2015). Providing introverts a meeting agenda ahead of time allows them to prepare to participate (Walter, 2013). They require time to consider information and should not be interrupted while speaking (Cooper, 2013). Introverts are successful in unpredictable environments (Grant et al., 2010); however, they are planners (Korn, 2017) who consider information before making decisions (Kuofie et al., 2015). When a quick decision is necessary, provide the reason and a timeframe whenever possible (Farrell, 2017).

\section{Create Flexible Workspaces}

Organizations can accommodate the needs of varying personality styles and foster happier employees (Kuofie et al., 2015). Approaches to productivity will differ between introverts and extroverts (Cooper, 2013); however, a comfortable environment increases everyone's productivity (Kuofie et al., 2015). Adaptability should go two ways with introverts and extroverts both giving and taking (Schmidt, 2016). Employees working in their preferred environment are most effective (Farrell, 2017), and constantly 


\section{Articles}

forcing someone into a different style hinders performance (Kuofie et al., 2015).

Organizations benefit from providing options to employees (Godsey, 2015). Extroverts need access to meeting areas for collaboration and brainstorming (Bernstein, 2015). Similarly, quiet spaces should be designated for introverts (Godsey, 2015). Constant stimulation from others overwhelms introverts (Grant, 2017; Remund, 2015). They require a proper outlet for processing information (Kuofie et al., 2015). This could simply be allowing introverts to use an empty conference room when concentration is necessary (Walter, 2013).

\section{Allow Schedule Creativity}

Personality style surfaces when individuals are faced with a new or unfamiliar task (Stewart, Et al., 2004). When possible, it is helpful to provide choices (Godsey, 2015). Introverts benefit from arranging schedules so draining events are not back-to-back (Korn, 2017). Employees need permission to find their balance between action and reflection (Remund, 2015) and build in alone time between taxing activities if necessary (Korn, 2017).

\section{Utilize the Strengths of Everyone}

Celebrating the unique traits of employees improves workplace performance (Kuofie et al., 2015). Organizations should foster the development of individual personality styles (Varvel et al., 2004). Leaders should examine organization needs to determine if extroverted or introverted traits are a better fit in specific situations (Farrell, 2017). Many expected gains from using a team approach have not been realized (Varvel et al., 2004). Everyone on a team needs a way to contribute ideas (Kuofie et al., 2015). Personality measure results allow organizations to build teams whose members complement one another (Varvel et al., 2004). Organizations, such as the Quiet Leadership Institute, provide workshops to help introverts be successful in the workplace and aid extroverts in managing introverts (Gordon, 2016).

\section{Conclusion}

Differing personality styles (Cooper, 2013) and subsequent tensions will be present in every workplace (Varvel, Adams, Pridie, \& Ulloa, 2004). While personality style differences are sometimes given too much significance, understanding and acknowledging differing preferences in the workplace will contribute to organization success (Gordon, 2016). It is important to remember available information on personality styles is generalization (Kuofie, Stephens-Craig, \& Dool, 2015) and will never fully describe a unique individual (Cooper, 2013). Leaders may take steps to ease workplace tensions created by personality style differences (Harold \& Holtz, 2015) and support individual employees in performing to their full potential (Kuofie et al., 2015). Further empirical research is needed to support common themes in popular culture and will assist organizations in sifting through the plethora of personality style information to determine what is truly valid and useful.

\section{References}

Abrams, A. (2017, June 25). Seven reasons to be proud to be an introvert. [Online]. Available: www.psychologytoday.com/blog/ nurturing-self-compassion/201706/sevenreasons-be-proud-be-introvert [19 July 2017].

Bakan, P., \& Leckart, B. T., 1966. Attention, extraversion, and stimulus-personality congruence. Perception and Psychophysics, pp.355-357.

Bernstein, E., 2015. Not introvert, nor extrovert: The adaptable ambivert. Wall Street Journal, pp. 1-3. 
Cain, S., 2012) Quiet: The power of introverts in a world that can't stop talking. New York, New York: Broadway Books.

Cooper, B. B. (2013, August 21). Are you an introvert or an extrovert? What it means for your career. [Online]. Available: www. fastcompany.com/3016031/are-you-anintrovert-or-an-extrovert-and-what-itmeans-for-your-career [19 July 2017].

Cullen-Lester, K. L., Leroy, H., Gerbasi, A., \& Nishii, L., 2016. Energy's role in the extraversion (dis)advantage: How energy ties and task conflict help clarify the relationship between extraversion and proactive performance. Journal of Organizational Behavior, pp. 1003-1022.

DiSalvo, D. (2013, April 21). Extroverts and introverts, make way for the ambiverts! [Online]. Available:

www.

p s y c holog y to d a y c o m / b log / neuronarrative/201304/extrovert-andintroverts-make-way-the-ambiverts [31 July 2017].

Dossey, L., 2016. Introverts: A defense. Explore: The Journal of Science and Healing, pp. 151-160.

Farrell, M., 2017. Leadership reflections: Extrovert and introvert leaders. Journal of Library Administration, pp. 436-443.

Glass, R., Li, S., \& Pan, R., 2014. Personality, problematic social network use, and academic performance in China. Journal of Computer Information Systems, pp. 88-96.

Godsey, M., 2015. When schools overlook introverts. The Atlantic, pp. 1-5.

Gordon, L. A., 2016. Introverts in an extroverts' world. American Bar Association Journal, pp. 36-41.

Grant, A. (2014, February 19). Myths about introverts and extraverts at work. [Online]. Available: www.huffingtonpost.com/adam- grant / 5 - m y th s - a b o u t introverts_b_4814390.html [31 July 2017].

Grant, A. M., 2013. Rethinking the extroverted sales ideal: The ambivert advantage. Psychological Science, pp. 1024-1030.

Grant, A. M., Gino, F., \& Hofmann, D. A., 2010. The hidden advantages of quiet bosses. Harvard Business Review, p. 28.

Grant, M. (2017, April 4). 5 myths about introverts that have been debunked by science. [Online]. Available: www.bustle. com/p/5-myths-about-introverts-that-havebeen-debunked-by-science-36143 [19 July 2017].

Harold, C. M., \& Holtz, B. C., 2015. The effects of passive leadership on workplace incivility. Journal of Organizational Behavior, pp. 16-38.

Huang, J. L., Bramble, R. J., Liu, M., Aqwa, J. J., Ott-Holland, C. J., Ryan, A. M., Wadlington, P. L., 2015. Rethinking the association between extraversion and job satisfaction: The role of interpersonal job context. Journal of Occupational and Organizational Psychology, pp. 683-691.

Hvidsten, A. K., 2016. Is introversion an obstacle in tacit knowledge sharing through socialization? Dalhousie Journal of Interdisciplinary Management, pp. 1-21.

Kasriel, S., 2016. How I did it...Upworks CEO on how an intoverted engineer learned to lead. Harvard Business Review, pp. 3538.

Korn, J. B., 2017. The dean as introvert. University of Toledo Law Review, pp. 297302.

Kuofie, M., Stephens-Craig, D., \& Dool, R., 2015. An overview perception of introverted leaders. International Journal of Global Business, pp. 93-103. 
Moore, K., \& McElroy, J. C., 2012. The influence of personality on facebook usage, wall postings, and regret. Computers in Human Behavior, pp. 267-274.

O'Brochta, M., 2014. Quiet leadership tips for project success. Roanoke, Virginia: Zozer, Inc.

Opt, S. K., \& Loffredo, D. A., 2000. Rethinking communication apprehension: A Myers-Briggs perspective. The Journal of Psychology, pp. 556-570.

Rauch, J., 2003. Caring for your introvert. The Atlantic Monthly, pp. 133-134.

Raza, A., Zaka-ul-Mustafa, \& Capretz, L. F., 2012. Do personality profiles differ in the Pakistani software industry and academia - A study. International Journal of Software Engineering, pp. 60-66.

Remund, D. L. (2015, March 2). Introverts in an extroverted profession. [Online]. Available: apps.prsa.org/Intelligence/ Tactics/Articles/view/10970/1106/ Introverts_in_an_Extroverted_Profession\#. Wa9QTnnXvIU [31 July 2017].

Sackett, P. R., \& Walmsley, P. T., 2014. Which personality attributes are most important in the workplace? Perspectives on Psychological Science, pp. 538-551.

Sadler-Smith, E., \& Badger, B., 1998. Cognitive style, learning, and innovation.
Technology Analysis and Strategic Management, pp. 247-265.

Schmidt, S., 2016. Personality diversity: Exrovert and introvert temperaments. Journal of Food Science Education, pp. 7374.

Skakoon, J. G., 2015. Introverts rule. Mechanical Engineering, p.1.

Stewart, B. L., Fritz, R. L., \& Norwood, M., 2003. Cognitive style as a component in meeting workplace demands for consumer services professionals: Benchmarking professional and student styles. Journal of Retailing and Consumer Services, pp. 279285.

Varvel, T., Adams, S. G., Pridie, S. J., \& Ulloa, B. C., 2004. Team effectiveness and individual Myers-Briggs personality dimensions. Journal of Management in Engineering, pp. 141-146.

Vien, C. L., 2016. Leadership tips for introverts. Journal of Accountancy, pp. 4750.

Walter, L. (2013, April 9). 5 tips to help introverts navigate work relationships. [Online]. Available: www.ehstoday.com/ safety/break-room-five-tips-help-introvertsnavigate-work-relationships [31 July 2017]. 